University of New Hampshire

University of New Hampshire Scholars' Repository

\title{
$5-2015$
}

\section{Probabilistic Reconstruction of Color for Species' Classification Underwater}

\author{
Yuri Rzhanov \\ University of New Hampshire, Durham, Yuri.Rzhanov@unh.edu \\ Shachak Pe'eri \\ University of New Hampshire, Durham, shachak.peeri@unh.edu
}

A. Shashkov

Klaipeda University

Follow this and additional works at: https://scholars.unh.edu/ccom

\section{Recommended Citation}

Y. Rzhanov, Pe'eri, S., and Shashkov, A., "Probabilistic Reconstruction of Color for Species' Classification Underwater", IEEE/MTS Oceans '15. Genova, Italy, 2015.

This Conference Proceeding is brought to you for free and open access by the Center for Coastal and Ocean Mapping at University of New Hampshire Scholars' Repository. It has been accepted for inclusion in Center for Coastal and Ocean Mapping by an authorized administrator of University of New Hampshire Scholars' Repository. For more information, please contact Scholarly.Communication@unh.edu. 


\section{Probabilistic reconstruction of color for species' classification underwater}

\author{
Yuri Rzhanov, Shachack Pe'eri \\ Center for Coastal and Ocean Mapping \\ University of New Hampshire \\ Durham, NH, USA \\ yuri.rzhanov@unh.edu
}

\author{
Aleksej Šaškov \\ Marine Science and Technology Center \\ Klaipeda University \\ Klaipeda, Lithuania
}

\begin{abstract}
Color is probably the most informative cue for object recognition and classification in natural scenes. Difference in shades can indicate to the biologist the potential for diversity of species or stress on the habitats. However, severe color distortions may occur in underwater imagery due to wavelengthdependent attenuation of light. Affordable tri-chromatic sensors are used to record the ambient light condition and color correct the imagery, but results show that this approach works reliably only under highly controllable conditions. This paper proposes an approach that combines hyperspectral data collected for the object of interest, hardware properties of the imaging sensor, and exterior conditions (optical properties of water and illumination) with tri-chromatic underwater imagery. Due to ambiguity of color reconstruction underwater, demonstrated in the paper, a probabilistic approach is used for classification that allows the identification of the object of interest from other objects.
\end{abstract}

Keywords-color underwater, spectrometry, benthic features, habitat classification

\section{INTRODUCTION}

Color is probably the one of the most informative cues for object (facies, micro-habitat, individual organism, etc.) recognition in underwater imagery. Color carries a wealth of useful information that can be used in a variety of marine applications - from health and stress levels of vegetation to identification of debris. However, many attempts to use color for underwater imagery directly were not very successful. In previous work, we have tried to assign specific palettes of colors to corresponding benthic features and to estimate their percentages of coverage on mosaics constructed from HD footage collected from an ROV platform [1,2]. Using photo mosaics allowed for inclusion of higher proportion of imagery in the analysis (each mosaic consisted on average of 750 frames), and thus derive better average cover values, and compensate for high heterogeneity of benthic environment.

Benthic features (especially biological ones) can rarely be defined by a single color, and some variability is always present. Therefore, a tolerance limit (threshold) should be applied even in perfect conditions, and several color palettes should be used to extract a single feature. Color palettes were chosen manually, by "trial and error" method until segmentation results on the test mosaics have become satisfactory. The main difficulty in the proposed approach is inconsistency of the imagery due to variation of the altitude of the acquisition platform, which is unavoidable in the "real world" underwater imagery. Although light attenuation in the water column can be considered constant during the acquisition, its effect on the benthic features colors during the image acquisition remains unknown. Therefore instead of reconstructing true colors, the mosaics were manually divided into color classes and separate sets of color palettes, independently created for each class. Even after that, segmentation results for each analyzed mosaic needed to be manually supervised, making it impossible to develop a fully automatic process.

The results obtained by this procedure were reasonably good for relatively uniform video mosaics, while for the mosaics with significant variations in colors results were much less successful (because of noticeable variations in acquisition platform altitude due to peculiarities of local bottom relief, ROV piloting mistakes, and hydrological factors affecting ROV movements underwater).

Contrary to the widespread opinion, it is not sufficient to "have a decent illumination and compensate for stronger absorption in the red part of the spectrum" to obtain a color which is reproducible and thus allows for reliable recognition of the object of interest (OoI).

The main reason for color restoration ambiguity is that objects usually have relatively complex spectral signatures (dependence of reflectivity on wavelength with changes that are on the order of tens of nanometers) and it is intuitively clear that just three measurements (R, G, and B channels with a spectral resolution of 70 to $150 \mathrm{~nm}$ ) are not sufficient to reconstruct original (as if imaged in air) color. Depending on current water properties, illumination, range to the target, intrinsic diversity of spectral signatures within the class of targets (benthic features) that need to be classified, and properties of the sensor (camera) itself, recorded color may vary in an amazingly wide range. This paper demonstrates the extent of color reconstruction ambiguity in a numerical experiment. To obtain a truly reproducible color measurement, the underwater platform must be equipped with a multispectral sensor which is expensive. Most platforms have affordable trichromatic sensors (color video and still cameras) and their data can be useful for OoI recognition on the condition that the spectral characteristics of the object are already known. In some cases the samples of OoI's can be taken to the top-side 
and the measurements done in the laboratory conditions [3]. It is more common to conduct the measurements in situ, which requires a specially built equipment. The design of a device for this purpose and results of preliminary tests are discussed below, as well as the plans for the future.

\section{COLOR RECONSTRUCTION AMBIGUity}

To demonstrate the color ambiguity it is sufficient to find two colors that appear visually different above water while providing exactly the same RGB components being observed underwater. However, it is interesting to obtain the distribution of "true" colors that collapse to a single color under the exactly same exterior conditions and sensor parameters. For this purpose a numerical experiment has been conducted. Calculating the colors observed underwater is a nonlinear process that involves integration over visible spectrum of a function depending on spectral properties of a light source, object reflectivity, light attenuation in the water column, and camera sensitivity function (e.g., [4]). A certain spectral signature for the observed object was chosen, and then trichromatic color at a known distance in water with given properties was calculated. Assuming the Lambertian shading model, pixel value recorded in color channel $f \in\{r, g, b\}$ is given by

$$
V_{f}=\int_{0}^{\infty} \omega I(\lambda) C(\lambda) \exp (-\kappa(\lambda) d) S_{f}(\lambda) d \lambda
$$

where $I(\lambda)$ is a light source, $S_{f}(\lambda)$ is the sensor quantum efficiency, $C(\lambda)$ is a OoI's reflectivity function (or spectral signature), $\kappa(\lambda)$ is water attenuation coefficient, $d$ is a light traveled distance, and $\omega$ is a constant describing camera gain and other settings. "True" pixel color is defined by the same expression without attenuation $(\kappa(\lambda)=0)$. To simplify calculations without loss of generality we chose constant light source $I(\lambda)=I_{0}$, Gaussian quantum efficiency curves

$$
S_{f}(\lambda)=q_{f} \exp \left(-\frac{\left(\lambda-\lambda_{f}\right)^{2}}{2 \sigma_{f}^{2}}\right)
$$

and quadratic dependence of absorption coefficient on wavelength

$$
\kappa(\lambda)=a_{k} \lambda^{2}+b_{k} \lambda+c_{k}=0.000015 \lambda^{2}-0.015 \lambda+3.8
$$

that gives minimum attenuation at $\lambda=500 \mathrm{~nm}, \kappa(500)=$ $0.05 \mathrm{~m}^{-1}, \kappa(700)=0.65 \mathrm{~m}^{-1}$. The latter closely resembles one of the Jerlov curves [5]. With all the above constants and dependencies fixed we have chosen the family of OoI's spectral signatures to be piecewise constant with three nonzero intervals. Thus, "true" and underwater color can be defined by 8 parameters: onset and offset wavelengths for non-zero intervals and their relative strengths (height ratios). Brightness of the result is not meaningful in this context, so each RGB triple is converted to CIE L*a*b* space, and all the color-related diagrams are shown in the $\left(* a,{ }^{*} b\right)$ space.

In general, the calculations have to be performed numerically. However, with this particular choice of spectral signatures the integration can be done explicitly using erf () function. The procedure is as follows. (1) Choose a certain set of 8 free parameters; $(2)$ calculate corresponding $(* a, * b)_{\mathrm{w}}$ and $(* a, * b)_{a}$ points for in-water and in-air ("true") cases respectively; and (3) using Monte Carlo technique, search and find another set of parameters which would lead to the same $\left(* a,{ }^{*} b\right)_{\mathrm{w}}$ but a different $\left(* a,{ }^{*} b\right)_{\mathrm{a}}$ point. Some striking examples are shown in Fig. 1.
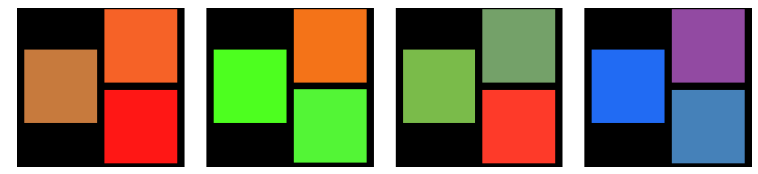

Figure 1. All colors have normalized brightness. Left squares: color recorded underwater. Right squares: colors recorded in air, appearing underwater as a corresponding left one.

Typical distribution of "true" colors corresponding to the same in-water color is shown in Fig. 2.

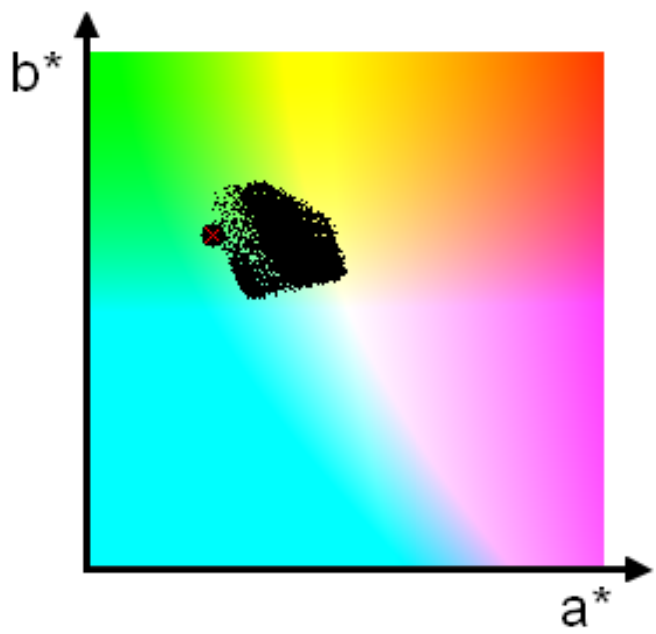

Figure 2. Distribution of $a^{*} b^{*}$ components of colors (black dots) leading to the same color recorded by a sensor underwater (marked by a large black dot with red slanted cross).

Obviously any change in exterior conditions or OoI's spectral signature will lead to a different distribution, so the one shown in Fig. 2 has only demonstrational value. It is not clear yet whether density of the distribution has any meaning (for example, of a probability density function (pdf)) or not, as the search has not been exhaustive.

\section{Proposed Approach To Use OF Underwater Color FOR CLASSIFICATION}

In order to avoid color ambiguity issue that may not allow to conduct proper classification, the following procedure is utilized. (1) Sensor (camera) and light source are calibrated in laboratory conditions. Ambient light spectrum and water properties are measured in situ (see, for example, [5]); (2) a representative number of spectral signatures of OoI is taken at the top side or in situ. Inherent variability provides for a family (catalogue) of spectral signatures which may depend on the season, geographical location, year, and etc.; (3) tri-chromatic imagery of an underwater scene is acquired in a standard way, and (4) the OoI catalogue is processed for the particular measured exterior conditions. This provides a real pdf in $\left(* a,{ }^{*} b\right)$ space. Each pixel in RGB imagery is compared with 
this pdf and the probability that it had imaged an OoI is estimated.

Various parameters, such as exterior conditions, sensor properties, range to the OoI, etc., strongly affect the recorded color. This fact plays an important role in ability to distinguish one OoI color family from another. Color families that are not distinguished under certain conditions (both map onto the same $\left(a^{*}, b^{*}\right)$ region), may become separable when multiple images are acquired at different ranges, using light sources with a different spectra, or by a different imaging sensors.

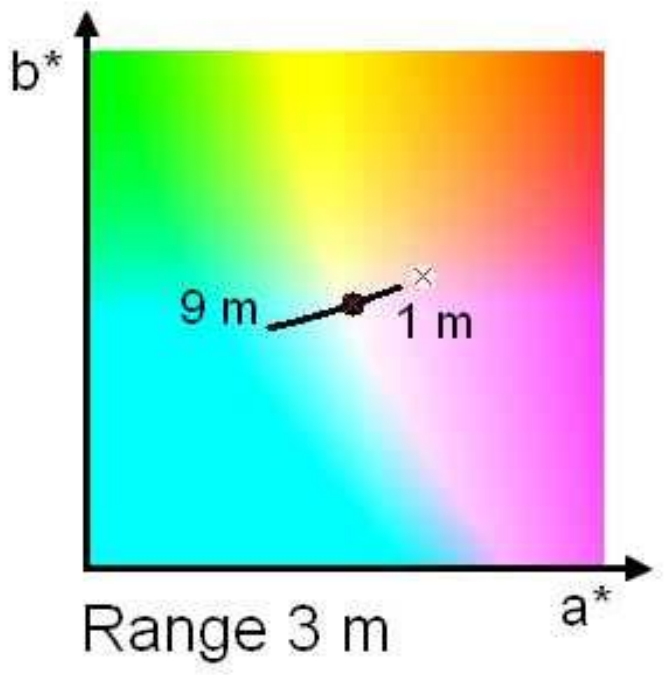

Figure 3. Disambiguation of OoI families by range variation. In air color obviously does not depend on range.

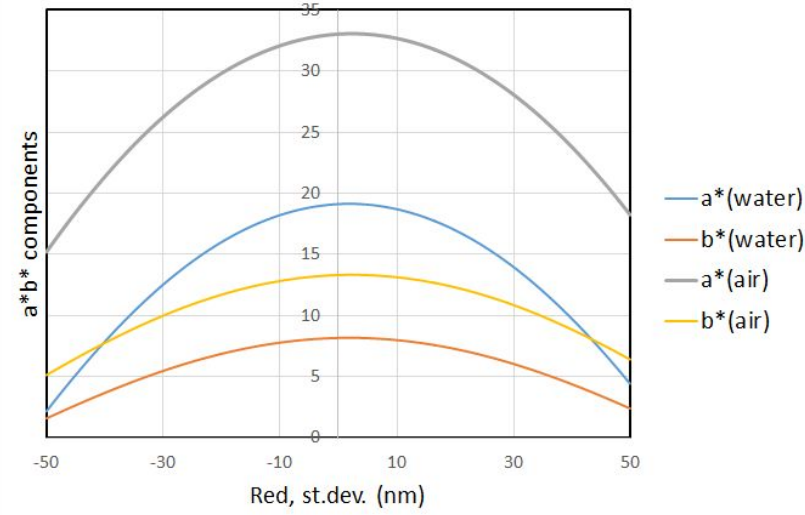

Figure 4. Disambiguation by sensor parameter variation.

Consider the $\left(a^{*}, b^{*}\right)$ chart shown in Fig.3. White dot with red slanted cross indicates "true" color. At a range of 3 meters the in-water color corresponds to the black dot with red slanted cross. However, increasing the range from 1 meter to 9 meters, the in-water color changes along the curve indicated $1 \mathrm{~m}-9 \mathrm{~m}$. Increasing some sensor parameter ${ }^{1}$ from $15 \mathrm{~nm}$ to $150 \mathrm{~nm}$ also leads to the change of in-water color shown in Fig.4. Thus, knowledge of OoI families allows for choice of conditions clearly separating in-water color clusters.

\footnotetext{
${ }^{1}$ Standard deviation of quantum efficiency curve for the red channel of the sensor $\sigma_{r}$.
}

\section{EXAMPLE OF REAL SPECTRA}

We have evaluated spectra of corals (Stylophora Pistillata): two samples from dead corals, D1 and D2, and two samples from healthy ones, L1 and L2, shown in Fig.5 [3].

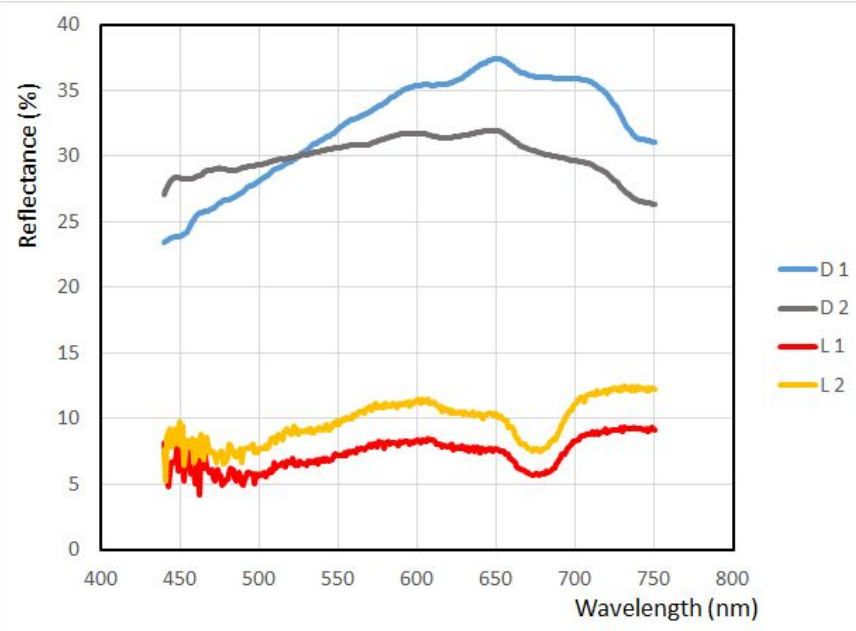

Figure 5. Corals' spectra acquired in the laboratory conditions.

Assuming certain sensor properties, illumination, and water conditions we may predict the color of the four coral samples at various ranges. Fig. 6 shows $\left(a^{*}, b^{*}\right)$ components of their colors. Note that color of the D1 sample is very similar to colors of healthy samples, as its spectrum is very close to that of healthy corals, with only reflectance level being different.

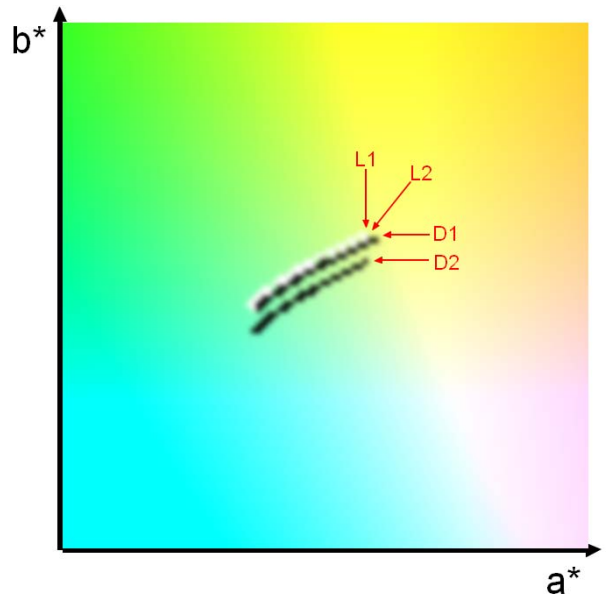

Figure 6. Color components for live and dead corals shown in a subregion of $\left(a^{*}, b^{*}\right)$ space.

\section{HARDWARE}

Hardware for measurement of water properties is available off-the-shelf. For example, accurate and effective measurement can be provided in situ by the HOBI Labs' a-Sphere (http://www.hobilabs.com). However, this is an expensive solution that may not be essential. Collection of water sample 
underwater and determination of water properties post factum would be sufficient. We have used a light source, quartz cuvette and spectrometer produced by Ocean Optics (http://oceanoptics.com). Ambient illumination at the time of acquisition of conventional imagery for classification can be measured by a spectrometer with a cosine corrector, to collect downwelling light. It is important to remember that the light that travels through water has two parts of the path: from water surface to the illuminated scene, and then to the camera. In some cases OoI spectra can be collected at the top side in the laboratory conditions. However, most of data must be collected in situ by a diver. For this purpose we have constructed a device which has a working name Underwater Recorder of Spectral Signatures (UROSS). The schematic drawing is presented in Fig. 7.

1

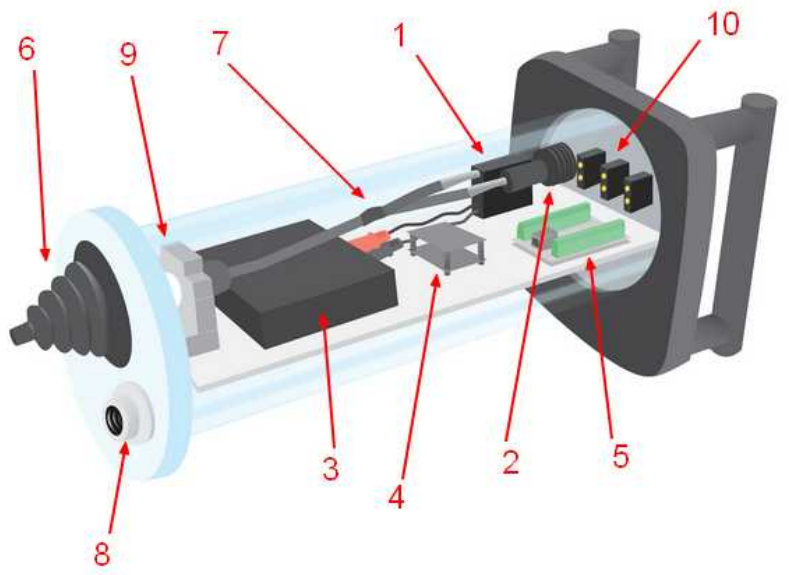

Figure 7. UROSS drawing.

The components are marked by numbers. The cables are not depicted for clarity of the sketch.

1. Spectrometer Qmini (http://www.rgb-lasers.com).

2. White broad-spectrum LED (http://immphotonics.de).

3. FitPC2 (http://www.fit-pc.com) running Windows 7.

4. Arduino Nano board communicating with a PC and controlling LCD display and temperature sensor (not shown).

5. USB trigger

(http://www.sensoriumembedded.com), converting signals from external switches to keystrokes for a PC.

6. Bellows for elimination of stray light during the measurement.

7. Custom-made bifurcated fiber optic cable transmitting the light from an LED to a collimator and reflected from OoI light to the spectrometer (http://www.thorlabs.com).

8. Low-range PC controlled still camera.

\section{Collimator.}

10. Three momentary switches and a power switch.

Battery pack (http://www.tenergy.com) (10000 mAh) is attached under the horizontal shelf and being fully charged provides sufficient power for four hours of non-stop work. Conventional frame camera in the front of the acquisition system is used as a reference for documentation to identify exactly which OoI spectrum that has been collected. Handles are attached to the back side of the device to allow a diver to hold and control the device using four switches. One to power the system and three momentary switches are used to send signals to a PC - to acquire a still image, to acquire spectrum signature, and to change folder in which the data are being saved.

Upon power-up, device's computer (FitPC2) turns on and automatically starts the UROSS software. A new root folder is created and a log file in which all actions (events) are recorded with appropriate timestamps. Within the root folder a data folder is created with all the recorded spectra and acquired images. When the user flicks "folder" switch, new sibling folder is created and all data files created after that are saved in this folder. This approach makes it easy to separate acquired data related to two or more OoI families.

The on-board computer has an HDMI connector allowing to upload the executable to Arduino and to debug the UROSS software responsible for acquisition and data logging when the water-proof housing is open. Setting up a wireless dedicated $a d$ hoc network allows to communicate with the computer and download data from it without taking the pressure housing off.

\section{TESTING}

Preliminary testing has shown that UROSS records reflectance well - up to 2500 measurements with a spectral range between 200 to $1000 \mathrm{~nm}$ at a $0.33 \mathrm{~nm}$ resolution. The measurements do demonstrate some noise which is attributed to a stray light. Binning of measurements at $5 \mathrm{~nm}$ (Fig.8) produces smooth spectral signatures with a stronger signal-tonoise ratio.

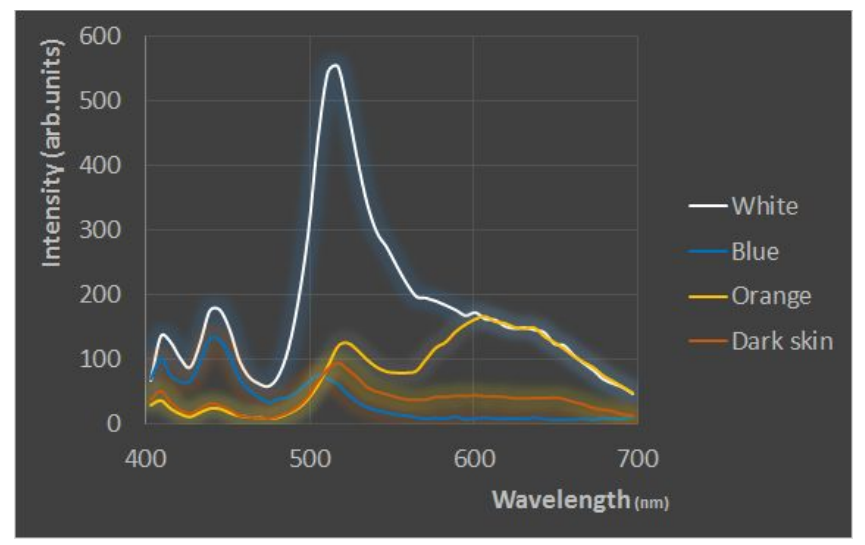

Figure 8. Spectra of four squares from the Macbeth chart. Exposure time $0.01 \mathrm{~s}$. 


\section{FUTURE WORK}

In the near future we plan to waterproof the housing of the system in order to withstand depths up to 30 meters and operate in different marine conditions around New England, USA (e.g., estuary, harbor, and offshore). We plan also to create a catalogue of spectral signatures for a number of OoI, specifically, macroalgal species, sediments, shell hash, and oyster beds. Conventional imagery acquired simultaneously will be processed and classified according to the scheme described in Section III. The results will be compared with the existing information and manual classification of images.

\section{ACKNOWLEDGMENTS}

This project was funded by the NOAA Joint Hydrographic Center grant NA05NOS4001153. The authors would like to thank Paul Lavoie and Carlo Lanzoni for help with hardware design and manufacturing.

\section{REFERENCES}

[1] A. Šaškov, T. G. Dahlgren, Y. Rzhanov, and M.-L. Schläppy, Comparing manual and semi-automatic underwater imagery analysis approaches for hard bottom benthic microfauna monitoring at offshore renewable energy installations, Hydrobiologia, doi: 10.1007/s10750014-2072-5, 2014.

[2] T. Dahlgren, M.-L. Schläppy, A. Shashkov, M,Andersson, Y. Rzhanov, and I. Fer, Assessing impact from wind farms at subtidal, exposed marine areas, chapter in Marine Renewable Energy Technology and Environmental Interactions, Humanity and the Sea. Springer Science, M.A. Shields, A.I.L. Payne (eds.), 2014.

[3] S.Pe'eri. Unpublished data.

[4] R.S. Berns, Principles of Color Technology, 3rd Ed., Wiley, New York (2001).
[5] https://water.usgs.gov/owq/FieldManual/Chapter6/Chapter6.0v2.pdf. Retrieved March $24^{\text {th }}, 2015$.

[6] C. Roelfsema et.al., Underwater Spectrometer System 2006 (UWSS04), Centre for Remote Sensing and Spatial Information Science for The University of Queensland, 2006. 